BONN-HE-93-25

DIAS-STP-93-13

July 1993

hep-th/9307190

\title{
The vacuum preserving Lie algebra of a classical $\mathcal{W}$-algebra
}

\author{
L. Fehér* \\ Physikalisches Institut der Universität Bonn \\ Nussallee 12, 53115 Bonn, Germany \\ L. O'Raifeartaigh and I. Tsutsui \\ Dublin Institute for Advanced Studies \\ 10 Burlington Road, Dublin 4, Ireland
}

\begin{abstract}
We simplify and generalize an argument due to Bowcock and Watts showing that one can associate a finite Lie algebra (the 'classical vacuum preserving algebra') containing the Möbius $\operatorname{sl}(2)$ subalgebra to any classical $\mathcal{W}$-algebra. Our construction is based on a kinematical analysis of the Poisson brackets of quasi-primary fields. In the case of the $\mathcal{W}_{\mathcal{S}}^{\mathcal{G}}$ algebra constructed through the Drinfeld-Sokolov reduction based on an arbitrary $\operatorname{sl}(2)$ subalgebra $\mathcal{S}$ of a simple Lie algebra $\mathcal{G}$, we exhibit a natural isomorphism between this finite Lie algebra and $\mathcal{G}$ whereby the Möbius $\operatorname{sl}(2)$ is identified with $\mathcal{S}$.
\end{abstract}

\footnotetext{
* An Alexander von Humboldt Fellow. On leave from Bolyai Institute of Szeged University, H-6720 Szeged, Hungary.
} 


\section{Introduction}

The classification of two dimensional conformal field theories (CFT) is an outstanding problem in theoretical physics, which is important for string theory, statistical physics, integrable systems, and various branches of mathematics. A subproblem to this is the problem of classifying the $\mathcal{W}$-algebras, that is the possible polynomial extensions of the chiral conformal algebra, the Virasoro algebra, by chiral primary fields. There is by now a large literature on this problem (see e.g. [1] and references therein), whose study was initiated by A. B. Zamolodchikov [2] by constructing unexpected examples of extended conformal algebras, in particular the $W_{3}$-algebra existing for continuously variable Virasoro centre. The classical version of the $W_{3}$-algebra was subsequently recognized [3] as the second Poisson bracket $(\mathrm{PB})$ algebra of the simplest generalized KdV hierarchy (the Boussinesq hierarchy), generalizing the relationship between the Virasoro algebra and the KdV hierarchy. Then it became clear that the second Poisson structures of the generalized KdV hierarchies constructed by means of a Hamiltonian reduction method by Drinfeld and Sokolov [4] all qualify as 'classical $\mathcal{W}$-algebras'. This led to extensive studies $[5,6,7]$ on the quantization of the Hamiltonian reductions of the affine Kac-Moody PB algebras considered by Drinfeld and Sokolov, and for a hunt after new $\mathcal{W}$-algebras and integrable systems obtained by generalizations of the Drinfeld-Sokolov construction.

Recently there has been remarkable progress in this field. First a classical $\mathcal{W}$-algebra was associated to each embedding of the Lie algebra $s l(2)$ into a simple Lie algebra [8] (see also [9]). This was done by a natural generalization of the Kac-Moody reduction considered by Drinfeld and Sokolov, using its interpretation in terms of the principal $s l(2)$ embedding found in [10]. More recently, an elegant quantization of this set of $\mathcal{W}$-algebras (called $\mathcal{W}_{\mathcal{S}}^{\mathcal{G}}$ algebras where $\mathcal{S} \subset \mathcal{G}$ is the $s l(2)$ embedding) was found [11,12] in the quantum Hamiltonian reduction (BRST) framework. At the same time, a number of arguments was put forward $[13]$ indicating that there are probably no other 'nice' $\mathcal{W}$-algebras that one could obtain in the Hamiltonian reduction framework since the $\mathcal{W}_{\mathcal{S}}^{\mathcal{G}}$-algebras appear to exhaust the $\mathcal{W}$ algebras resulting from Drinfeld-Sokolov type reduction. For example, it was shown that the spectrum of conformal weight $\Delta \geq \frac{3}{2}$ generators in any such $\mathcal{W}$-algebra must always be the same as in a corresponding $\mathcal{W}_{\mathcal{S}}^{\mathcal{G}}$-algebra.

The above results on the construction and classification of $\mathcal{W}$-algebras were obtained in the Hamiltonian reduction approach, but there exists also a more abstract argument pointing to the crucial rôle of $s l(2)$ embeddings in this context. This is due to Bowcock and Watts [14], who studied the classification of 'reductive' quantum $\mathcal{W}$-algebras. These are operator product algebras (meromorphic conformal field theories [15]) generated by a Virasoro field and a finite set of chiral primary fields with half-integral conformal weights 
$\Delta \geq 1$ that exist for a continuous range of the Virasoro centre $C$ and admit a classical $(C \rightarrow \infty)$ limit (see [14] for precise definition). The corresponding classical $\mathcal{W}$-algebra is then a PB algebra of fields $W^{a}(z)(a=1, \ldots, N)$ on the circle $S^{1}(|z|=1)$ of the type

$$
\left\{W^{a}(z), W^{b}(w)\right\}=\sum_{k \geq 0} P_{k}^{a b}\left(W^{1}(w), \ldots, W^{N}(w)\right) \partial_{z}^{k} \delta(z-w),
$$

where the sum is finite, the $P_{k}^{a b}(W)$ 's are differential polynomials or constants, and one of the fields is a Virasoro density with respect to which the others are primary fields. The idea of Bowcock and Watts [14] is to consider the space $\tilde{\mathcal{G}}$ of 'vacuum preserving Fourier modes' given by

$$
\tilde{\mathcal{G}}:=\operatorname{span}\left\{W_{m}^{a}|| m \mid \leq\left(\Delta_{a}-1\right)\right\},
$$

and define a Lie algebra structure on $\tilde{\mathcal{G}}$ by truncating $(0.1)$ to its linear part (for the Fourier mode convention, see (1.7)). Then they showed that this procedure yields a finite Lie algebra by using the commutator formula $[16,17]$ of chiral quasi-primary fields in meromorphic CFT. The importance of the construction derives from the fact that $\tilde{\mathcal{G}}$ automatically contains the Möbius $s l(2)$ subalgebra $\tilde{\mathcal{S}}$ spanned by the vacuum preserving modes of the Virasoro. This immediately implies that the conformal spectrum of the $\mathcal{W}$-algebra is encoded in the spin content of the decomposition of $\tilde{\mathcal{G}}$ into $\tilde{\mathcal{S}}$ irreps (which are simply the vacuum preserving modes of the fields). This means that the possible conformal spectra are determined by the possible $s l(2)$ embeddings into finite Lie algebras, and since it was also argued [14] that the finite Lie algebra $\tilde{\mathcal{G}}$ is necessarily a reductive (semisimple + abelian) Lie algebra the $s l(2)$ embeddings are given by Dynkin's list [18].

The main purpose of the present note is to point out that the above argument actually applies to the classification of classical $\mathcal{W}$-algebras directly, without viewing them as the classical limit of a quantum $\mathcal{W}$-algebra as was done in [14]. By using only some simple kinematical properties of Poisson brackets, we shall show that one can associate the finite Lie algebra $\tilde{\mathcal{G}}$ (the 'classical vacuum preserving algebra') to any classical $\mathcal{W}$-algebra. This means for example that the result about the possible conformal spectra applies directly to the classification of classical $\mathcal{W}$-algebras, and then 'a fortiori' to the quantum $\mathcal{W}$-algebras having a classical limit. Our purely classical derivation is not only a simplification, but in this way we also obtain a certain generalization of the Bowcock-Watts result since we do not use various, not always satisfied, properties of an underlying meromorphic CFT required in [14] (e.g. the positivity and integral conformal weight assumptions). After presenting this, in Section 2 we shall consider the $\mathcal{W}_{\mathcal{S}}^{\mathcal{G}}$-algebras and show that the 'vacuum preserving data' $(\tilde{S}, \tilde{\mathcal{G}})$ can in this case be naturally identified with the 'Drinfeld-Sokolov data' $(\mathcal{S}, \mathcal{G})$. This result was derived in [14] in the integral weight case. The derivation we present is different, and we shall also cover the half-integral case. 


\section{Finite Lie algebra from PB algebra of quasi-primary fields}

Suppose that we have some finite set of quasi-primary fields $W^{a}(z)$ defined on the circle $S^{1}$, with half-integral weights $\Delta_{a} \geq 1$ with respect to an action of the Möbius group implemented through Poisson bracket:

$$
\begin{aligned}
\left\{L_{m}, W^{a}(z)\right\} & =z^{m}\left(z \partial_{z}+(m+1) \Delta_{a}\right) W^{a}(z), \\
\left\{L_{m}, L_{n}\right\} & =(m-n) L_{m+n}, \quad m, n=0, \pm 1 .
\end{aligned}
$$

Suppose that the PB's of the fields $W^{a}(z)$ close in a local, differential polynomial way. More precisely, suppose that we have

$$
\left\{W^{a}(z), W^{b}(w)\right\}=g_{a b} \delta^{\left(\Delta_{a}+\Delta_{b}-1\right)}+\sum_{c} \sum_{n=0}^{\Delta(a b c)-1} A_{c}^{a b}(n)\left(\partial^{n} W^{c}\right)(w) \delta^{(\Delta(a b c)-1-n)}+\cdots
$$

where the dots stand for terms quadratic and higher order in the $W$ 's; the summation is on those $c$ for which $\Delta(a b c):=\Delta_{a}+\Delta_{b}-\Delta_{c} \in \mathbf{N} ; \delta^{(m)}:=\partial_{z}^{m} \delta(z-w)$; and if $\left(\Delta_{a}+\Delta_{b}\right) \notin \mathbf{N}$ then $g_{a b}=0$. We are interested in the coefficients $g_{a b}$ and $A_{c}^{a b}(n)$. By taking the PB of eq. (1.2) with the Möbius generator $L_{1}$ using (1.1), and comparing the two sides of the resulting equation up to linear terms in the $W^{\prime}$ 's, one obtains that $g_{a b}$ can be nonzero only for fields of equal weights,

$$
g_{a b}=g_{a b} \delta_{\Delta_{a}, \Delta_{b}}
$$

and also obtains a recursion relation for the $A_{c}^{a b}(n)$. This leads to the following result:

$$
A_{c}^{a b}(n)=\frac{\left(1+\Delta_{c}-\Delta_{a}-\Delta_{b}\right)_{(n)}\left(\Delta_{a}-\Delta_{b}+\Delta_{c}\right)_{(n)}}{n !\left(2 \Delta_{c}\right)_{(n)}} A_{c}^{a b}(0),
$$

where for any $x \in \mathbf{C}$ and $n \in \mathbf{N}$ we define

$$
(x)_{(n)}:=\prod_{m=0}^{n-1}(x+m), \quad \text { and } \quad(x)_{(0)}:=1
$$

The next step is to express (1.2) in Fourier modes by using eqs. (1.3-4) together with

$$
\oint d z f(z) \delta(z-w)=f(w), \quad \delta(z-w)=\frac{1}{2 \pi i} \sum_{m \in \mathbf{Z}} z^{m} w^{-(m+1)}
$$

and the modes defined by

$$
W^{a}(z):=\sum_{m \in \mathbf{Z}+\Delta_{a}} W_{m}^{a} z^{-m-\Delta_{a}}
$$


One then finds the following formula:

$$
\begin{aligned}
\left\{W_{m}^{a}, W_{n}^{b}\right\}= & \frac{(-1)^{2 \Delta_{a}-1}}{2 \pi i}\left(\prod_{k=-\left(\Delta_{a}-1\right)}^{\left(\Delta_{a}-1\right)}(m+k)\right) g_{a b} \delta_{\Delta_{a}, \Delta_{b}} \delta_{m,-n} \\
& +\sum_{\{c: \Delta(a b c) \in \mathbf{N}\}} B_{c}^{a b} P\left(m, n ; \Delta_{a}, \Delta_{b}, \Delta_{c}\right) W_{m+n}^{c} \cdots
\end{aligned}
$$

with

$$
B_{c}^{a b}:=\frac{(-1)^{\Delta(a b c)-1}}{2 \pi i}(\Delta(a b c)-1) ! A_{c}^{a b}(0), \quad \Delta(a b c)=\Delta_{a}+\Delta_{b}-\Delta_{c} \geq 1
$$

and

$$
\begin{aligned}
& P\left(m, n ; \Delta_{a}, \Delta_{b}, \Delta_{c}\right)= \\
& \quad \sum_{r=0}^{\Delta(a b c)-1} \frac{(-1)^{r}}{r !}\left(\begin{array}{c}
m+\Delta_{a}-1 \\
\Delta_{a}+\Delta_{b}-\Delta_{c}-1-r
\end{array}\right) \frac{\left(\Delta_{a}-\Delta_{b}+\Delta_{c}\right)_{(r)}\left(m+n+\Delta_{c}\right)_{(r)}}{\left(2 \Delta_{c}\right)_{(r)}} .
\end{aligned}
$$

As an aside, we should at this point note that the same polynomials $P$ in (1.10) appear in the commutator formula of quasi-primary fields in the quantum case $[16,17]$, simply because they express purely kinematical information. To derive the commutator formula one could proceed in the quantum case similarly as above, by demanding the covariance of the operator product of quasi-primary fields under the Möbius group. This derivation appears simpler than the ones given in refs. [16,17], which inspired the above consideration.

We now consider the 'linearized vacuum preserving PB algebra' (vpa for short*) defined by

$$
\left[W_{m}^{a}, W_{n}^{b}\right]:=\sum_{c} B_{c}^{a b} P\left(m, n ; \Delta_{a}, \Delta_{b}, \Delta_{c}\right) W_{m+n}^{c}, \quad|m| \leq\left(\Delta_{a}-1\right),|n| \leq\left(\Delta_{b}-1\right)
$$

i.e., we drop the quadratic and higher order terms on the r.h.s. of (1.8). It is not hard to see that this formula provides a PB on the 'vp modes' $\left\{W_{m}^{a}\right\},|m| \leq\left(\Delta_{a}-1\right)$. Indeed, if $W_{m}^{a}$ and $W_{n}^{b}$ are vp modes but $W_{m+n}^{c}$ is not so, i.e., $|m+n|>\left(\Delta_{c}-1\right)$, then

$$
P\left(m, n ; \Delta_{a}, \Delta_{b}, \Delta_{c}\right)=0
$$

This follows from the fact that

$$
P\left(m, n ; \Delta_{a}, \Delta_{b}, \Delta_{c}\right) \sim C_{m n}^{j_{a} j_{b} j_{c}} \quad \text { for } \quad|m| \leq j_{a},|n| \leq j_{b}
$$

* Although there is obviously no vacuum in the classical case we keep the terminology vpa to maintain contact with previous work [14]. 
where $j_{a}:=\Delta_{a}-1$ (etc.), and $C_{m n}^{j_{a} j_{b} j_{c}}$ is the standard Clebsch-Gordan coefficient of $s u(2)$. This was verified in [17] from the explicit formula (1.10). A noncomputational argument yielding (1.12-13) would be to combine the fact that the vp modes of a quasi-primary field carry a finite dimensional irreducible representation (irrep) of the Möbius $\operatorname{sl}(2)$ with the fact that the set of such irreps is stable under tensor products, and the PB of the vp modes behaves like a tensor product when acted on by the Möbius generators. (We sketched the derivation of (1.10) above since it contains more information.) After establishing that only vp modes appear on the r.h.s. of (1.11), one easily sees that the Jacobi identity is satisfied for the linearized PB (1.11) as a consequence of the Jacobi identity of the original PB (1.8) on account of the absence of central term between a vp mode and an arbitrary other mode in (1.8). In conclusion, one can associate the finite dimensional Lie algebra $(\tilde{\mathcal{G}},[]$,$) given$ by $(0.2),(1.11)$ to any PB algebra of fields of the type given by (1.1-2).

In particular, if one of the fields in (1.2) is the Virasoro density whose vp modes are the Möbius modes $\left\{L_{-1}, L_{0}, L_{1}\right\}$, then the vpa $\tilde{\mathcal{G}}$ automatically comes equipped with the preferred $\operatorname{sl}(2)$ subalgebra $\tilde{\mathcal{S}}:=\operatorname{span}\left\{L_{-1}, L_{0}, L_{+1}\right\}$. In this case, by construction $\tilde{\mathcal{G}}$ decomposes under $\tilde{\mathcal{S}}$ into the direct sum of the spin $j_{a}:=\left(\Delta_{a}-1\right)$ irreps spanned by the vp modes of the $W^{a}$. This means that the conformal spectrum $\Delta_{a}$ of the generating fields $W^{a}(z)$ of a classical $\mathcal{W}$-algebra is always determined by an $\operatorname{sl}(2)$ decomposition of a finite Lie algebra and, a fortiori, the same can be said about those quantum $\mathcal{W}$-algebras that admit a classical limit [14]. Thus the question of classifying the possible conformal spectra of such $\mathcal{W}$-algebras reduces to the classification of $\operatorname{sl}(2)$ embeddings, which is known if the vpa is a reductive (semisimple+abelian) Lie algebra [18]. Moreover, $\mathcal{W}$-algebras with any such 'in principle possible' conformal spectrum can actually be easily constructed by Drinfeld-Sokolov reduction.

\section{The vpa of $\mathcal{W}_{\mathcal{S}}^{\mathcal{G}}$ is $\mathcal{G}$}

Let $\mathcal{G}$ be a finite dimensional simple Lie algebra and $\mathcal{S}=\operatorname{span}\left\{M_{-}, M_{0}, M_{+}\right\} \subset \mathcal{G}$ an $\operatorname{sl}(2)$ subalgebra,

$$
\left[M_{0}, M_{ \pm}\right]= \pm M_{ \pm}, \quad\left[M_{+}, M_{-}\right]=2 M_{0}
$$

To this data one can associate a natural $\mathcal{W}$-algebra, designated as $\mathcal{W}_{\mathcal{S}}^{\mathcal{G}}$, and construct its vpa along the lines of the previous section. This gives a finite Lie algebra $\tilde{\mathcal{G}}$, endowed with the Möbius embedding $\tilde{\mathcal{S}} \subset \tilde{\mathcal{G}}$. The $\operatorname{sl}(2)$ spins in the decomposition of $\mathcal{G}$ under $\mathcal{S}$ coincide with those in the decomposition of $\tilde{\mathcal{G}}$ under $\tilde{\mathcal{S}}$ since both sets of $\operatorname{sl}(2)$ spins is related to the set of conformal weights of $\mathcal{W}_{\mathcal{S}}^{\mathcal{G}}$ by a shift by 1 (as is obvious for $\tilde{S} \subset \tilde{\mathcal{G}}$ and well known for $\mathcal{S} \subset \mathcal{G})$. Thus one expects $(\mathcal{S}, \mathcal{G})$ to be isomporphic to $(\tilde{\mathcal{S}}, \tilde{\mathcal{G}})$. Indeed, this has been shown by Bowcock and Watts [14] in the case of an integral $s l(2)$ embedding, by a rather involved 
computation. The purpose of this section is to give a simple proof covering half-integral $s l(2)$ embeddings as well. We first recall the definition of the $\mathcal{W}_{\mathcal{S}}^{\mathcal{G}}$-algebra $[8,9]$.

The $\mathcal{W}_{\mathcal{S}}^{\mathcal{G}}$-algebra is a reduction of the 'KM Poisson bracket algebra' constructed as follows. We first consider the space $\mathcal{K}$ of $\mathcal{G}$-valued smooth functions ('currents') $J(z)$ defined on the circle $S^{1}$. We let the space $\mathcal{K}$ carry the Poisson bracket

$$
\{\langle\alpha, J(z)\rangle,\langle\beta, J(w)\rangle\}=\langle[\alpha, \beta], J(z)\rangle \delta(z-w)+\langle\alpha, \beta\rangle \delta^{\prime}(z-w), \quad \alpha, \beta \in \mathcal{G},
$$

where $\langle$,$\rangle is the invariant scalar product on \mathcal{G}$. For our purpose it is useful to introduce the 'shifted current' $j(z):=J(z)-M_{-}$in terms of which (2.2) reads

$$
\{\langle\alpha, j(z)\rangle,\langle\beta, j(w)\rangle\}=(\langle[\alpha, \beta], j(z)\rangle+\omega(\alpha, \beta)) \delta(z-w)+\langle\alpha, \beta\rangle \delta^{\prime}(z-w),
$$

where $\omega$ is the 2 -form on $\mathcal{G}$ given by $\omega(\alpha, \beta):=\left\langle M_{-},[\alpha, \beta]\right\rangle$. We then impose the constraints

$$
\phi_{\tau_{i}}(z):=\left\langle\tau_{i}, j(z)\right\rangle=0
$$

where $\left\{\tau_{i}\right\}$ is a basis of the subspace $\left[M_{+}, \mathcal{G}\right] \subset \mathcal{G}$. The corresponding constraint surface is the affine subspace $\mathcal{K}_{\mathrm{hw}} \subset \mathcal{K}$ consisting of currents of the following $(\operatorname{sl}(2)$ highest weight) form

$$
J(z)=M_{-}+j_{\mathrm{hw}}(z), \quad j_{\mathrm{hw}}(z) \in \operatorname{Ker}\left(\operatorname{ad}_{M_{+}}\right) .
$$

The constraints (2.4) are second class and the $\mathcal{W}_{\mathcal{S}}^{\mathcal{G}}$-algebra is just the Dirac bracket algebra carried by the components of the constrained current $j_{\mathrm{hw}}(z)$. It is convenient to choose a basis $\left\{\sigma^{a, m}\right\} \subset \mathcal{G}$ adapted to the decomposition of $\mathcal{G}$ under $\mathcal{S}$. The index a runs over the $s l(2)$ irreps and for fixed $a$ we have $-l_{a} \leq m \leq l_{a}$, where $l_{a}$ is the spin of the irrep, with the normalization fixed according to

$$
\sigma^{a, m}=\left(\operatorname{ad}_{M_{-}}\right)^{l_{a}-m}\left(\sigma^{a, l_{a}}\right), \quad\left[M_{0}, \sigma^{a, m}\right]=m \sigma^{a, m} .
$$

Defining $W^{a}(z):=\left\langle\sigma^{a,-l_{a}}, j_{\mathrm{hw}}(z)\right\rangle$, the Dirac bracket of our interest is

$$
\begin{aligned}
& \left\{W^{a}(z), W^{b}(w)\right\}^{*}=\left(\left\{\left\langle\sigma^{a,-l_{a}}, j(z)\right\rangle,\left\langle\sigma^{b,-l_{b}}, j(w)\right\rangle\right\}\right. \\
& \left.\quad-\sum_{i, k} \oint \oint d u d v\left\{\left\langle\sigma^{a,-l_{a}}, j(z)\right\rangle, \phi_{\tau_{i}}(u)\right\} D_{i k}^{-1}(u, v ; j)\left\{\phi_{\tau_{k}}(v),\left\langle\sigma^{b,-l_{b}}, j(w)\right\rangle\right\}\right) \mid \mathcal{K}_{\mathrm{hw}},
\end{aligned}
$$

where $D_{i k}^{-1}(u, v ; j)$ is the inverse of $D_{i k}(u, v ; j):=\left\{\phi_{\tau_{i}}(u), \phi_{\tau_{k}}(v)\right\}$, i.e.,

$$
\sum_{k} \oint d u D_{i k}(z, u ; j) D_{k m}^{-1}(u, w ; j)=\delta_{i m} \delta(z-w) .
$$


Eq. (2.7) defines a differential polynomial algebra since $D_{i k}^{-1}(u, v ; j)$ is a linear combination of $\delta(u-v)$ and its derivatives with coefficients that are differential polynomials in $j(u)$. The field $W^{a}(z)$ is quasi-primary with weight $\Delta_{a}=l_{a}+1$ with respect to the Virasoro density

$$
L(z)=\left\langle M_{-}, j_{\mathrm{hw}}(z)\right\rangle+\frac{1}{2}\left\langle j_{\mathrm{hw}}(z), j_{\mathrm{hw}}(z)\right\rangle
$$

whose two terms are commuting Virosoro densities (under the Dirac bracket). Notice also that the Virasoro (2.9) is the restriction of the Sugawara formula to the constraint surface $(2.5)$.

Having recalled the definition of $\mathcal{W}_{\mathcal{S}}^{\mathcal{G}}$, we are now in a position to study its vpa $(\tilde{\mathcal{G}},[]$,$) ,$ which is obtained (cf. (1.8) and (1.11)) by linearizing (2.7) as

$$
\left\{W_{m}^{a}, W_{n}^{b}\right\}^{*}:=\left[W_{m}^{a}, W_{n}^{b}\right]+\mathcal{O}\left(j_{\mathrm{hw}}^{2}\right)
$$

for the vp modes

$$
W_{m}^{a}:=\frac{1}{2 \pi i} \oint d z W^{a}(z) z^{m+l_{a}}, \quad-l_{a} \leq m \leq l_{a}
$$

and similarly for $b$. In $(2.10) \mathcal{O}\left(j_{\text {hw }}^{2}\right)$ denotes the quadratic and higher order terms in the Dirac $(\mathcal{W}$-algebra) bracket of the vp modes. We shall prove that the following correspondence,

$$
\sigma^{a, m} \Longleftrightarrow \widehat{W}_{m}^{a}, \quad \text { with } \quad \widehat{W}_{m}^{a}:=\frac{2 \pi i}{\left(m+l_{a}\right) !} W_{m}^{a}
$$

is an isomorphism between the original Lie algebra $\mathcal{G}$ and the vpa $\tilde{\mathcal{G}}$,

$$
\left[\sigma^{a, m}, \sigma^{b, n}\right] \Longleftrightarrow\left[\widehat{W}_{m}^{a}, \widehat{W}_{n}^{b}\right]
$$

We start by noting that, for any test function $f(z)$, we have the $\mathcal{W}$-charge

$$
W_{f}^{a}\left[j_{\mathrm{hw}}\right]:=\oint d z\left\langle\sigma^{a,-l_{a}}, j_{\mathrm{hw}}(z)\right\rangle f(z)
$$

which is a function on $\mathcal{K}_{\text {hw }}$ (the vp modes (2.11) being special cases). We then define the corresponding 'improved $\mathcal{W}$-charge'

$$
\begin{aligned}
\widetilde{W}_{f}^{a}[j]:= & \oint d z\left\langle\sigma^{a,-l_{a}}, j(z)\right\rangle f(z) \\
& -\sum_{i, k} \oint \oint \oint d u d v d z f(z)\left\{\left\langle\sigma^{a,-l_{a}}, j(z)\right\rangle, \phi_{\tau_{i}}(u)\right\} D_{i, k}^{-1}\left(u, v ; j_{\mathrm{hw}}\right) \phi_{\tau_{k}}(v),
\end{aligned}
$$


which is a function on $\mathcal{K}$ that reduces to $W_{f}^{a}$ on $\mathcal{K}_{\mathrm{hw}}$ and (weakly) commutes with the constraints,

$$
\left.\left\{\widetilde{W_{f}^{a}}, \phi_{\tau_{\mathrm{i}}}(z)\right\}\right|_{\mathcal{K}_{\mathrm{hw}}}=0
$$

(For clarity, we note that $j_{\mathrm{hw}}$ in the argument of $D^{-1}$ in (2.14) denotes the projection of $j$ on $\operatorname{Ker}\left(\operatorname{ad}_{M_{+}}\right)$, given by the decomposition $\left.\mathcal{G}=\left[M_{-}, \mathcal{G}\right]+\operatorname{Ker}\left(\operatorname{ad}_{M_{+}}\right)\right)$. Since $\widetilde{W}_{f}^{a}[j]$ is a differential polynomial in $j$, we can separate its linear part, denoted by $\mathcal{L}_{f}^{a}[j]$, as

$$
\widetilde{W}_{f}^{a}[j]=\mathcal{L}_{f}^{a}[j]+\mathcal{O}\left(j^{2}\right)
$$

We shall see below that the information contained in the linearized Dirac bracket giving the vpa by (2.10) can be extracted from the linear terms of the improved $\mathcal{W}$-charges. Taking the linear part of (2.14) yields

$$
\begin{aligned}
\mathcal{L}_{f}^{a}[j] & :=\oint d z\left\langle\sigma^{a,-l_{a}}, j(z)\right\rangle f(z) \\
& -\sum_{i, k} \oint \oint \oint d u d v d z f(z)\left\{\left\langle\sigma^{a,-l_{a}}, j(z)\right\rangle, \phi_{\tau_{i}}(u)\right\}_{j=0} D_{i, k}^{-1}\left(u, v ; j_{\mathrm{hw}}=0\right) \phi_{\tau_{k}}(v),
\end{aligned}
$$

from which we find the following explicit formula:

$$
\mathcal{L}_{f}^{a}[j]=\sum_{k=0}^{2 l_{a}} \oint d z\left\langle\sigma^{a, k-l_{a}}, j(z)\right\rangle \partial^{k} f(z) .
$$

As an alternative to inspecting the kernel $D_{i k}^{-1}\left(u, v ; j_{\mathrm{hw}}=0\right)$, the reader can verify this formula by checking that it satisfies

$$
\left\{\mathcal{L}_{f}^{a}, \phi_{\tau_{\mathrm{i}}}(z)\right\}_{j=0}=0
$$

which is a consequence of eqs. (2.15-16).

Equation (2.18) is the key to our considerations since we now show that it implies the required isomporhism $(2.12)$. For this we first note that, because of the identity $W_{f}^{a}\left[j_{\mathrm{hw}}\right]=$ $\widetilde{W}_{f}^{a}\left[j_{\mathrm{hw}}\right]$ and $(2.15)$, we have

$$
\left\{W_{f_{1}}^{a}, W_{f_{2}}^{b}\right\}^{*}\left[j_{\mathrm{hw}}\right]=\left\{\widetilde{W}_{f_{1}}^{a}, \widetilde{W}_{f_{2}}^{b}\right\}\left[j_{\mathrm{hw}}\right]
$$

for any two test functions $f_{1}, f_{2}$. In other words, we can use the original PB instead of the Dirac bracket if we replace the $\mathcal{W}$-charges by their 'improved' versions. (A similar trick is always available, at least locally, when dealing with a Dirac bracket.) On the other hand, we have

$$
\left\{\mathcal{L}_{f}^{a}, \mathcal{O}\left(j^{2}\right)\right\}[j]=\mathcal{O}\left(j^{2}\right), \quad \text { for } \quad f(z) \sim z^{m+l_{a}} \quad \text { with } \quad|m| \leq l_{a}
$$


as is readily verified from (2.18) using (2.3). By combining (2.20) with (2.16) and (2.21), we see that the linearized bracket $\left[W_{m}^{a}, W_{n}^{b}\right]$ defined in $(2.10)$ is the same as the original KacMoody $\mathrm{PB}$ of the linear terms of the corresponding improved $\mathcal{W}$-charges. More precisely, we have

$$
\left[\widehat{W}_{m}^{a}, \widehat{W}_{n}^{b}\right]=\left\{\mathcal{L}_{m}^{a}, \mathcal{L}_{n}^{b}\right\}\left[j_{\mathrm{hw}}\right]
$$

where we used the notation introduced in $(2.12 \mathrm{a})$ and defined

$$
\mathcal{L}_{m}^{a}:=\mathcal{L}_{f}^{a} \quad \text { with } \quad f=\frac{z^{m+l_{a}}}{\left(m+l_{a}\right) !} .
$$

Observe that one can rewrite $\mathcal{L}_{m}^{a}$ given by (2.18) and (2.23) as

$$
\mathcal{L}_{m}^{a}[j]=\oint d z\left\langle\widehat{\sigma}^{a, m}, j(z)\right\rangle
$$

where

$$
\widehat{\sigma}^{a, m}:=g \sigma^{a, m} g^{-1} \text { with } g:=e^{z M_{-}} .
$$

From this and (2.3) it follows that if in the $\mathcal{S}$-basis $\mathcal{G}$ is given by

$$
\left[\sigma^{a, m}, \sigma^{b, n}\right]=\sum_{c, k} f_{(c, k)}^{(a, m),(b, n)} \sigma^{c, k}
$$

then we have

$$
\left\{\mathcal{L}_{m}^{a}, \mathcal{L}_{n}^{b}\right\}[j]=\sum_{c, k} f_{(c, k),(b, n)}^{(a, m),(b, c} \mathcal{L}_{k}^{c}[j] .
$$

Combining this with $(2.22)$ and using that $\mathcal{L}_{k}^{c}\left[j_{\mathrm{hw}}\right]=\widehat{W}_{k}^{c}$, we conclude that (2.12) gives indeed the claimed isomorphism between the vpa of $\mathcal{W}_{\mathcal{S}}^{\mathcal{G}}$ and $\mathcal{G}$ :

$$
\left[\widehat{W}_{m}^{a}, \widehat{W}_{n}^{b}\right]=\sum_{c, k} f_{(c, k)}^{(a, m),(b, n)} \widehat{W}_{k}^{c} .
$$

Under the isomorphism (2.12) the $\operatorname{sl}(2)$ subalgebra $\mathcal{S}=\operatorname{span}\left\{M_{-}, M_{0}, M_{+}\right\} \subset \mathcal{G}$ corresponds to the $\operatorname{sl}(2)$ spanned by the vp modes of $\left\langle M_{-}, j_{\mathrm{hw}}\right\rangle$, the first term in (2.9). At the linearized level this $\operatorname{sl}(2)$ is the same as the Möbius $\operatorname{sl}(2)$ spanned by the vp modes of the full Virasoro density $L$ in (2.9), and thus we have recovered the $s l(2)$ embedding data used in the construction of the $\mathcal{W}_{\mathcal{S}}^{\mathcal{G}}$-algebra from its linearized vacuum preserving algebra.

We finish by noting that it is an interesting open question whether a classical $\mathcal{W}$-algebra is completely determined by its vpa and centre in general.

Acknowledgements. L.F. wishes to thank H. Arfaei, A. Honecker and W. Nahm for discussions and for comments on the manuscript. He is also grateful to the Alexander von Humboldt-Stiftung for support. 


\section{References}

[1] P. Bouwknegt and K. Schoutens, Phys. Rep. 223 (1993) 183.

[2] A. B. Zamolodchikov, Theor. Math. Phys. 65 (1985) 1205.

[3] K. Yamagishi, Phys. Lett. 205B (1988) 466;

P. Mathieu, Phys. Lett. 208B (1988) 101;

I. Bakas, Phys. Lett. 213B (1988) 313.

[4] V. G. Drinfeld and V. V. Sokolov, Jour. Sov. Math. 30 (1984) 1975.

[5] V. A. Fateev and S. L. Lukyanov, Int. J. Mod. Phys. A3 (1988) 507; Sov. Sci. Rev. A Phys. 15 (1990) 1.

[6] M. Bershadsky and H. Ooguri, Commun. Math. Phys. 126 (1989) 49.

[7] B. L. Feigin and E. Frenkel, Phys. Lett. 246B (1990) 75;

E. Frenkel, V. G. Kac and M. Wakimoto, Commun. Math. Phys. 147 (1992) 295.

[8] F. A. Bais, T. Tjin and P. van Driel, Nucl. Phys. B357 (1991) 632.

[9] L. Fehér, L. O’Raifeartaigh, P. Ruelle, I. Tsutsui and A. Wipf, Phys. Rep. 222 (1992) 1.

[10] J. Balog, L. Fehér, P. Forgács, L. O’Raifeartaigh and A. Wipf, Ann. Phys. (N. Y.) 203 (1990) 76.

[11] J. de Boer and T. Tjin, preprint THU-92-32, IFTA-28-92, hep-th/9211109; preprint THU-93/05, IFTA-02-93, hep-th/9302006.

[12] A. Sevrin and W. Troost, preprint LBL-34125, UCB-PTH-93/19, KUL-TF-93/21, hepth/9306033.

[13] L. Fehér, L. O’Raifeartaigh, P. Ruelle and I. Tsutsui, preprint BONN-HE-93-14, DIASSTP-93-02, hep-th/9304125.

[14] P. Bowcock and G. M. T. Watts, Nucl. Phys. B379 (1992) 63.

[15] P. Goddard, in: Infinite Dimensional Lie Algebras and Lie Groups, ed. V. G. Kac, World Scientific, 1989.

[16] W. Nahm, in: Proc. Trieste Conf. on Recent Developments in Conformal Field Theory, Trieste, October 1989; Conformal Quantum Field Theories in Two Dimensions, World Scientific, to appear.

[17] P. Bowcock, Nucl. Phys. B356 (1991) 367.

[18] E. B. Dynkin, Amer. Math. Soc. Transl. 6 [2] (1957) 111. 